\title{
A CONVERGENCE FACTOR THEOREM IN THE THEORY OF SUMMABLE SERIES*
}

BY H. L. GARABEDIAN

1. Introduction. The object of this paper is to write down sufficient conditions to ensure that any definition of summability of the convergence factor type $\dagger$ be more effective than or include $\ddagger$ the definition of de la Vallée-Poussin.

A series

$$
\sum_{n=0}^{\infty} u_{n}=u_{0}+u_{1}+u_{2}+\cdots
$$

is said to be summable by the method of de la Vallee-Poussin (or summable $V P$ ) to the sum $S$ if $\lim _{n \rightarrow \infty} v_{n}=S$, where

$$
\begin{aligned}
v_{n} & =u_{0}+\sum_{k=1}^{n} \frac{C_{2 n, n-k}}{C_{2 n, n}} u_{k} \\
& =u_{0}+\sum_{k=1}^{n} \frac{n(n-1) \cdots(n-k+1)}{(n+1)(n+2) \cdots(n+k)} u_{k} .
\end{aligned}
$$

For the sake of convenience, we shall define the series (1) to be $\phi$-summable to the sum $S$ provided that the set of functions, $\phi_{n}(\alpha),(n=0,1,2, \cdots)$, are defined for a set of values $E(\alpha)$ having at least one limit point $\alpha_{0}$, not of the set; the series $\sum_{n=0}^{\infty} \phi_{n}(\alpha) u_{n}$ converges over $E(\alpha)$; and

$$
\lim _{\alpha \rightarrow \alpha_{0}} \sum_{n=0}^{\infty} \phi_{n}(\alpha) u_{n}=S .
$$

The theorem studied in this paper is stated as follows.

Theorem. If (i) the series (1) is summable VP to the sum $S$, (ii) the set of functions, $\phi_{n}(\alpha),(n=0,1,2, \cdots)$, defined for a set of values $E(\alpha)$ having at least one limit point $\alpha_{0}$, not of the set, satisfies the conditions

* Presented to the Society, September 5, 1934.

† C. N. Moore, Transactions of this Society, vol. 8 (1907), p. 299.

$\ddagger$ W. A. Hurwitz, this Bulletin, vol. 28 (1922), p. 17. 
(a $\operatorname{aim}_{1} \lim _{\alpha \rightarrow \alpha_{0}} \phi_{n}(\alpha)=1, \quad\left(\mathrm{a}_{2}\right) \quad \lim _{n \rightarrow \infty} \nu^{n} \phi_{n}(\alpha)=0$, over $E(\alpha)$,

(a) $\quad \sum_{n=0}^{\infty} C_{2 n, n}\left|\sum_{\lambda=0}^{\infty} C_{\lambda+2 n, 2 n} \Delta^{2 n+2} \phi_{2 \lambda+n}(\alpha)\right|<K$, over $E(\alpha)$,

where $\nu$ and $K$ are independent of $\alpha$ and $n$, and $\nu>e^{5}$, (iii)* $\phi$-summability includes Cesàro summability of arbitrary integral order; then the series $\sum_{n=0}^{\infty} \phi_{n}(\alpha) u_{n}$ converges over $E(\alpha)$, and

$$
\lim _{\alpha \rightarrow \alpha_{0}} \sum_{n=0}^{\infty} \phi_{n}(\alpha) u_{n}=S \text {. }
$$

We conclude this introduction with the remark that the raison d'être of this theorem may be traced to a somewhat similar theorem, due to Bromwich, $\dagger$ in which sufficient conditions are given in order that $\phi$-summability include $(C k)$ summability. It will be observed that an analogy can be drawn between the conditions of the present theorem and those of the Bromwich theorem despite the discrepancy in complexity of the problems involved.

2. Proof of Theorem. At the outset we shall consider the de la Vallée-Poussin matrix and its inverse, denoted respectively by $(V P)$ and $(V P)^{-1}$, and their use in satisfying some of the exigencies of the problem at hand.

The matrix $(V P)$ is of triangular type, that is,

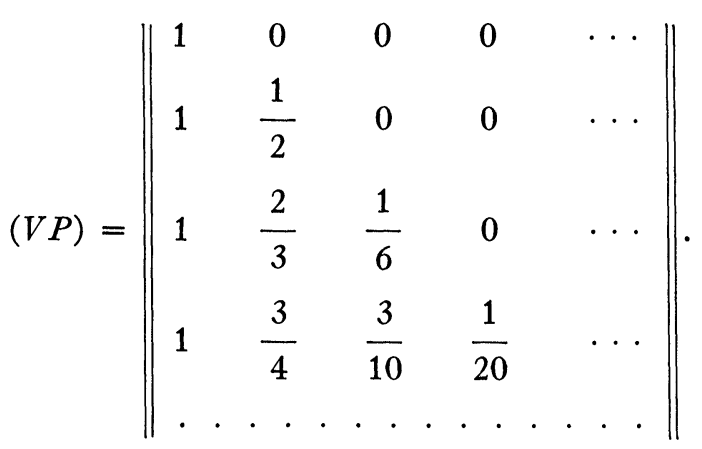

* Let us recall that $V P$ summability includes $(C k)$ summability, that is, Cesàro summability of order $k$. This result was obtained independently and virtually simultaneously by T. H. Gronwall, Comptes Rendus, vol. 158 (1914), p. 1664, and C. N. Moore, Comptes Rendus, vol. 158 (1914), p. 1774.

$\dagger$ Mathematische Annalen, vol. 65 (1907-08), p. 351. 
The inverse matrix $(V P)^{-1}$ is known to be*

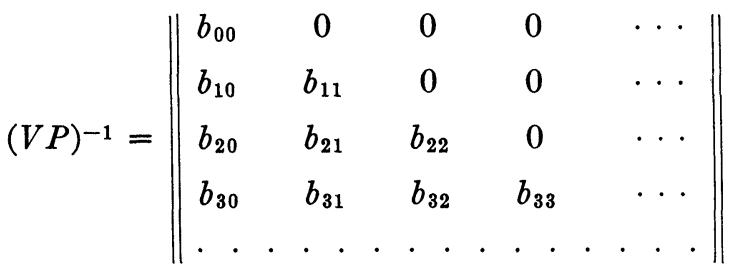

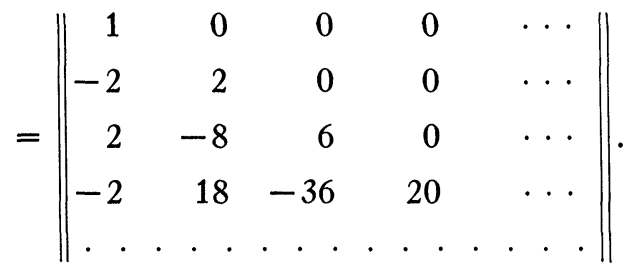

The matrix $(V P)^{-1}$ gives the solution of the equations (2) for the $u$ 's in terms of the $v$ 's:

(3) $u_{0}=v_{0}, \quad u_{n}=2 \sum_{k=0}^{n}(-1)^{n-k} C_{n, k} C_{n+k-1, k} v_{k}$,

$$
(n=1,2,3, \cdots) .
$$

Note that

$$
\begin{aligned}
b_{00}=1, b_{n k}=2(-1)^{n-k} C_{n, k} C_{n+k-1, k}, & \\
& (k=0,1,2, \cdots, n ; n \neq 0) .
\end{aligned}
$$

Form the series

$$
\sum_{n=0}^{\infty} \phi_{n}(\alpha) u_{n}
$$

and replace $u_{n}$ in (4) by its value in (3) to get

$$
\sum_{n=0}^{\infty} \sum_{k=0}^{n} b_{n k} \phi_{n}(\alpha) v_{k} .
$$

At this juncture, we find it expedient to pause temporarily to prove the following lemma.

* George Rutledge, Journal of Mathematics and Physics, M. I. T., vol. 11 (1932), p. 76. 
LEMMA 1.

$$
\sum_{n=0}^{\infty} \sum_{k=0}^{n} b_{n k} \phi_{n}(\alpha) v_{k}=\sum_{n=0}^{\infty} \sum_{k=n}^{\infty} b_{k n} \phi_{k}(\alpha) v_{n} .
$$

The right-hand member of (6) is obtained from (5) by an interchange in the order of limits. In order to prove this identity we must establish completely the validity of the infinite processes involved.

We find it convenient to write out one of the members of (6) in the form of a double series:

$$
\begin{aligned}
& b_{00} \phi_{0} v_{0}+0+0+0+0+\ldots \\
& +b_{10} \phi_{1} v_{0}+b_{11} \phi_{1} v_{1}+0+0+0 \\
& +b_{20} \phi_{2} v_{0}+b_{21} \phi_{2} v_{1}+b_{22} \phi_{2} v_{2}+0+\ldots \\
& +b_{30} \phi_{3} v_{0}+b_{31} \phi_{3} v_{1}+b_{32} \phi_{3} v_{2}+b_{33} \phi_{3} v_{3}+0 \text {. } 0+\ldots \\
& +. . \text {. . . . . . . . . . . . . . . . . }
\end{aligned}
$$

The left member of equation (6) represents the double series (7) summed by rows, and the right-hand member of (6) represents the series (7) summed by columns. We wish to show that the sums obtained in these two different fashions are identical. Now, it follows from a theorem due to Pringsheim* that if the rows and columns of a double series converge, and if the double series is convergent, then the interchange of limits implied in (6) is permissible. We shall first show that the individual rows and the individual columns of the series (7) converge, and secondly execute the more difficult task of showing that the double series itself converges.

The individual rows of (7) converge since the terms are all zero from a certain point on. The individual columns are series of the form $\sum_{k=n}^{\infty} \phi_{k}(\alpha) b_{k n} v_{n},(n=0,1,2, \cdots)$, or

$$
\left\{\begin{array}{l}
2 \sum_{k=n}^{\infty}(-1)^{k-n} C_{k, n} C_{k+n-1, n} \phi_{k}(\alpha) v_{n}, \\
{\left[\phi_{0}+2 \sum_{k=1}^{\infty}(-1)^{k} \phi_{k}(\alpha)\right] v_{0} .}
\end{array}\right.
$$

\footnotetext{
* Bromwich, Theory of Infinite Series, 1st or 2d edition, $\$ 30$.
} 
We have $b_{k n}=O\left[(k+n) ! /(n !)^{2}(k-n) !\right]$. Using Stirling's well known formula, for the $\Gamma$-function, we can write

$$
b_{k n}=O\left[(k+n)^{k+n} /\left\{n^{2 n}(k-n)^{k-n}\right\}\right] .
$$

Now, set $k=n+m$, where $m$ is a positive integer, and we obtain

$$
b_{k n}=O\left[(2 n+m)^{2 n+m} /\left\{n^{2 n} m^{m}\right\}\right]=O\left[4^{n} e^{m} e^{2 n}\right] .
$$

Substituting $m=k-n$ in this last expression we can write

$$
b_{k n} \phi_{k}=O\left[4^{n} e^{k+n} \phi_{k}\right]=O\left[e^{k+3 n} \phi_{k}\right]=O\left[e^{4 k} \phi_{k}\right] .
$$

From condition $\left(\mathrm{a}_{2}\right)$ of the hypotheses we have $\phi_{k}=O\left(\nu^{-k}\right)$, where $\nu>e^{5}$. Consequently, it follows from (10) that

$$
b_{k n} \phi_{k}=O\left(e^{-k}\right) \text {. }
$$

The equation (11) implies the convergence of the series (8) and hence the convergence of the individual columns.

Let us now form the sum, $s_{p q}$, of the $p q$ terms taken from the first $p$ rows and the first $q$ columns of the series (7). The convergence of $s_{p q}$ as $p$ and $q$ tend to infinity in such a manner that $p \leqq q$ follows from the existence of the left-hand side of (6). Note that the existence of this expression is in turn implied by the existence of the series (4). To establish the convergence of the series (4) we recall first of all that, since $\lim _{k \rightarrow \infty} v_{k}$ exists, $v_{k}$ is bounded. Then, from (9) and the discussion above it follows that $b_{n k} v_{k}=O\left(e^{n+3 k}\right), u_{n}=\sum_{k=0}^{n} b_{n k} v_{k}=O\left(e^{4 n}\right)$ and $\phi_{n} u_{n}=O\left(e^{-n}\right)$. This suffices to prove the convergence of the series (4). For the case $p>q$ we must show that the additional terms included are negligible. This additional block of terms is

$$
\begin{gathered}
b_{q, 0} \phi_{q} v_{0}+b_{q, 1} \phi_{q} v_{1}+\cdots+b_{q, q} \phi_{q} v_{q} \\
+b_{q+1,0} \phi_{q+1} v_{0}+b_{q+1,1} \phi_{q+1} v_{1}+\cdots+b_{q+1, q} \phi_{q+1} v_{q} \\
+\cdot \cdot \cdot \cdot \cdot \cdot \cdot \cdot \cdot \cdot \cdot \cdot \cdot \cdot \cdot \cdot \cdot \cdot \cdot \cdot \\
+b_{p, 0} \phi_{p} v_{0}+b_{p, 1} \phi_{p} v_{1}+\cdots+b_{p, q} \phi_{p} v_{q} .
\end{gathered}
$$

Designate an arbitrary one of these terms by $b_{i j} \phi_{i} v_{j}$, $i=q, q+1, \cdots, p ; \quad j=0,1, \cdots, q ; \quad p \geqq i \geqq q ; \quad q \geqq j \geqq 0$. We have

$$
b_{i j} \phi_{i} v_{j}=2(-1)^{i-j} C_{i, j} C_{i+j-1, j} \phi_{i} v_{j} .
$$


From (11) and the boundedness of $v_{j}$ it follows that

$$
b_{i j} \phi_{i} v_{i}=O\left(e^{-i}\right) .
$$

The equation (1.3) implies that the sum of the terms $b_{i j} \phi_{i} v_{j}$ of the expression (12) can be made small enough to be negligible by taking $q$ sufficiently large. In turn, this implies the convergence of $s_{p q}$. Accordingly, we have established the validity of the identity (6).

Now, to return to our main argument, it follows from (4), (5), and the lemma above that we can set $F(\alpha)=\sum_{n=0}^{\infty} \phi_{n}(\alpha) u_{n}$ and write

$$
F(\alpha)=\sum_{n=0}^{\infty} c_{\alpha n} v_{n}, \quad c_{\alpha n}=\sum_{k=n}^{\infty} \phi_{k}(\alpha) b_{k n} .
$$

Since $\lim _{n \rightarrow \infty} v_{n}=S$, it remains to show that the method of summation, of the convergence factor type, defined by (14) is regular, ${ }^{*}$ which is to say that $\lim _{\alpha_{\rightarrow} \alpha_{0}} F(\alpha)=S$. Accordingly, it is required in the present case that

$$
\lim _{\alpha \rightarrow \alpha_{0}} c_{\alpha n}=0 \text { for every } n,
$$

$K$ independent of $\alpha$.

$$
\lim _{\alpha \rightarrow \alpha_{0}} \sum_{n=0}^{\infty} c_{\alpha n}=1 \text {, }
$$

It remains to prove that the conditions (b) are fulfilled whenever the conditions of the theorem are satisfied. Recall that

$$
c_{\alpha 0}=\phi_{0}+2 \sum_{k=1}^{\infty}(-1)^{k} \phi_{k}(\alpha),
$$

and, for $n=1,2, \cdots$,

$$
c_{\alpha n}=2 \sum_{k=n}^{\infty}(-1)^{k-n} C_{k, n} C_{k+n-1, n} \phi_{k}(\alpha),
$$

and notice that, since $b_{k n}=0,(k<n)$,

$$
c_{\alpha n}=\sum_{k=n}^{\infty} \phi_{k}(\alpha) b_{k n}=\sum_{k=0}^{\infty} \phi_{k}(\alpha) b_{k n} .
$$

\footnotetext{
* Hurwitz, loc. cit., p. 20.
} 
At this point, the following identity* proves to be of fundamental importance:

$$
\sum_{k=0}^{\infty} b_{k n} x^{k}=(1-x)^{2 n+2} \sum_{k=0}^{\infty} S_{k}^{(2 n+1)} x^{k}, \quad(|x|<1),
$$

where

$$
\begin{aligned}
S_{k}^{(2 n+1)}= & C_{2 n+k+1,2 n+1} b_{0 n}+C_{2 n+k, 2 n+1} b_{1 n} \\
& +C_{2 n+k-1,2 n+1} b_{2 n}+\cdots+C_{2 n+1,2 n+1} b_{k n} .
\end{aligned}
$$

It results at once from (18) that

$$
b_{k n}=S_{k}^{(2 n+1)}-C_{2 n+2,1} S_{k-1}^{(2 n+1)}+C_{2 n+2,2} S_{k-2}^{(2 n+1)}
$$

$$
-\cdots+(-1)^{2 n+2} C_{2 n+2,2 n+2} S_{k-2 n-2}^{(2 n+1)},
$$

where it is understood that when a negative subscript occurs in the formula the corresponding $S^{(2 n+1)}$ is to be replaced by zero. Substitute the value for $b_{k n}$ in (19) in the equation (17) to get

$$
\begin{aligned}
c_{\alpha n}= & \sum_{k=0}^{\infty}\left[S_{k}^{(2 n+1)}-C_{2 n+2,1} S_{k-1}^{(2 n+1)}+C_{2 n+2,2} S_{k-2}^{(2 n+1)}\right. \\
& \left.-\cdots+(-1)^{2 n+2} C_{2 n+2,2 n+2} S_{k-2 n-2}^{(2 n+1)}\right] \phi_{k}(\alpha) .
\end{aligned}
$$

Ordering the terms of (20) with respect to $S_{k}^{(2 n+1)}$, we obtain formally

$$
\begin{aligned}
c_{\alpha n} & =\sum_{k=0}^{\infty} S_{k}^{(2 n+1)}\left[\phi_{k}(\alpha)-C_{2 n+2,1} \phi_{k+1}(\alpha)+\cdots\right. \\
& \left.+(-1)^{r} C_{2 n+2, r} \phi_{k+r}(\alpha)+\cdots+(-1)^{2 n+2} \phi_{k+2 n+2}(\alpha)\right],
\end{aligned}
$$

or

$$
\text { (21) } \quad c_{\alpha n}=\sum_{k=n}^{\infty} S_{k}^{(2 n+1)} \Delta^{2 n+2} \phi_{k}(\alpha),
$$

where we notice that $S_{k}^{(2 n+1)}=0,(k<n)$. In order to justify this

\footnotetext{
* Bromwich, loc. cit., 1st ed., p. 311.
} 
last step it will suffice to establish the absolute convergence of the series

$$
\sum_{k=i}^{\infty} S_{k-i}^{(2 n+1)} \phi_{k}(\alpha), \quad(i=0,1,2, \cdots, 2 n+2) .
$$

To this end and for further reference we delay once again to prove a fundamental lemma.

LEMMA 2.

$$
\begin{aligned}
S_{k}^{(2 n+1)}=C_{2 n, n} & C_{\lambda+2 n, 2 n}, \\
& (k=2 \lambda+n ; n=1,2, \cdots) .
\end{aligned}
$$

From equation (18) we have the formula

$$
\begin{aligned}
& \sum_{k=0}^{\infty} S_{k}^{(2 n+1)} x^{k} \\
& \quad=2(1-x)^{-(2 n+2)} \sum_{k=0}^{\infty}(-1)^{k-n} C_{k, n} C_{k+n-1, n} x^{k},
\end{aligned}
$$

which is needed later in the proof.

Multiplying the binomial expansion of $(1+x)^{-(2 n+1)}$ by $C_{2 n, n} x^{n}(1-x)$, we obtain

$$
\begin{aligned}
C_{2 n, n} & \frac{x^{n}(1-x)}{(1+x)^{2 n+1}} \\
\quad= & \frac{2}{(n !)^{2}} \sum_{j=0}^{\infty}(-1)^{j}(n+j)(2 n+j-1) \cdots(j+1) x^{j+n} .
\end{aligned}
$$

Multiplying both sides of $(25)$ by $(1-x)^{-(2 n+2)}$ and setting $j+n=k$ in the right member, we get

$$
C_{2 n, n} x^{n}\left(1-x^{2}\right)^{-(2 n+1)}
$$

$$
=2(1-x)^{-(2 n+2)} \sum_{k=0}^{\infty}(-1)^{k-n} C_{k, n} C_{n+k-1, n} x^{k} .
$$

Now, eliminating the expression on the right-hand side of (26) between formulas (23) and (26), we have 


$$
\begin{aligned}
\sum_{k=0}^{\infty} S_{k}^{(2 n+1)} x^{k} & =C_{2 n, n} x^{n}\left(1-x^{2}\right)^{-(2 n+1)} \\
& =C_{2 n, n} \sum_{\lambda=0}^{\infty} C_{\lambda+2 n, 2 n} x^{2 \lambda+n} .
\end{aligned}
$$

Our lemma follows at once from this identity.

From (22) and (23) we have

$$
\begin{aligned}
\sum_{k=i}^{\infty} S_{k-i}^{(2 n+1)} \phi_{k}(\alpha) & =\sum_{m=0}^{\infty} S_{m}^{(2 n+1)} \phi_{m+i}(\alpha) \\
& =C_{2 n, n} \sum_{\lambda=0}^{\infty} C_{\lambda+2 n, 2 n} \phi_{2 \lambda+n+i}(\alpha) .
\end{aligned}
$$

Moreover, it is easy to prove the formulas $C_{\lambda+2 n, 2 n}=O\left(e^{\lambda}\right)$ and $\phi_{2 \lambda+n+i}(\alpha)=O\left[e^{-(2 \lambda+i)}\right]$. Consequently,

$$
C_{\lambda+2 n, 2 n} \phi_{2 \lambda+n+i}(\alpha)=O\left[e^{-(\lambda+i)}\right]
$$

and the absolute convergence of the series (22) is assured.

Now, formulas (21) and (23) are used to eliminate $S_{k}(2 n+1)$ and obtain

$$
c_{\alpha n}=C_{2 n, n} \sum_{\lambda=0}^{\infty} C_{\lambda+2 n, 2 n} \Delta^{2 n+2} \phi_{2 \lambda+n}(\alpha), \quad(n=1,2, \cdots) .
$$

Furthermore, it is easily proved from (15) that

$$
c_{\alpha 0}=-\phi_{0}(\alpha)+2 \sum_{k=0}^{\infty}(-1)^{k} \phi_{k}(\alpha)=\sum_{\lambda=0}^{\infty} \Delta^{2} \phi_{2 \lambda}(\alpha) .
$$

Accordingly, formula (27) also holds for $n=0$.

We are now prepared to discuss the conditions (b), first of all $\left(b_{1}\right)$. Since the series $\sum_{k=0}^{\infty}(-1)^{k}$ is summable $(C 1)$ to the value $1 / 2$, and since, by condition (iii) of the hypotheses, $\phi$-summability includes ( $C 1)$ summability, it follows from (28) that $\lim _{\alpha \rightarrow \alpha_{0}} c_{\alpha 0}=0$. Consequently, the condition $\left(b_{1}\right)$ is satisfied for $n=0$. Let us now focus our attention on the series

$$
\sum_{k=n}^{\infty} C_{k, n} C_{k+n-1, n}(-1)^{k-n}, \quad(n=1,2, \cdots) .
$$


If we form the Cesàro mean of order $2 n+1$ for this series and use formula (23), we have

$$
\begin{aligned}
\lim _{k \rightarrow \infty} & S_{k}^{(2 n+1)} / C_{k+2 n+1,2 n+1} \\
& =\lim _{\lambda \rightarrow \infty} C_{2 n, n} C_{\lambda+2 n, 2 n} / C_{\lambda+4 n+1,2 n+1}=0, \quad(n=1,2, \cdots) .
\end{aligned}
$$

Accordingly, the series in question is summable $(C, 2 n+1)$ to the value zero. It follows a fortiori from formula (16) that $\lim _{\alpha \rightarrow \alpha_{0}} c_{\alpha n}=0,(n=1,2, \cdots)$. This completes the proof that condition $\left(b_{1}\right)$ is satisfied for all values of $n$.

Let us consider condition $\left(b_{2}\right)$. We find from formula (17) that

$$
\sum_{n=0}^{\infty} c_{\alpha n}=\sum_{n=0}^{\infty} \sum_{k=n}^{\infty} \phi_{k}(\alpha) b_{k n} .
$$

An interchange in the order of summation in the double series (29) gives

$$
\sum_{n=0}^{\infty} c_{\alpha n}=\sum_{n=0}^{\infty} \phi_{n}(\alpha) \sum_{k=0}^{n} b_{n k} .
$$

This operation is justified by the identity obtained by equating the $v$ 's to unity in Lemma 1 . It is quite evident from the matrix $(V P)^{-1}$ that $\sum_{k=0}^{n} b_{n k}=0,(n=1,2, \cdots)$, and this can readily be proved by complete induction. Moreover, $b_{00}=1$. It follows from equation (30) that $\sum_{n=0}^{\infty} c_{\alpha n}=\phi_{0}(\alpha)$, and hence that $\lim _{\alpha \rightarrow \alpha_{0}} \sum_{n=0}^{\infty} c_{\alpha n}=1$. Accordingly, condition $\left(b_{2}\right)$ has been shown to be satisfied.

Finally, it follows from (27) and the subsequent discussion that

$$
\sum_{n=0}^{\infty}\left|c_{\alpha n}\right|=\sum_{n=0}^{\infty} C_{2 n, n}\left|\sum_{\lambda=0}^{\infty} C_{\lambda+2 n, 2 n} \Delta^{2 n+2} \phi_{2 \lambda+n}(\alpha)\right|,
$$

and this expression is clearly uniformly bounded, over $E(\alpha)$, provided condition $\left(a_{3}\right)$ obtains. Thus, the last of the conditions (b) has been shown to be satisfied. This completes the proof of our theorem.

NORTHWESTERN UNIVERSITY 